\title{
Myocardial Characterization in Patients with Nonischemic Dilated Cardiomyopathy Combined with Ventricular Arrhythmias: Insights from Cardiovascular Magnetic Resonance Feature Tracking
}

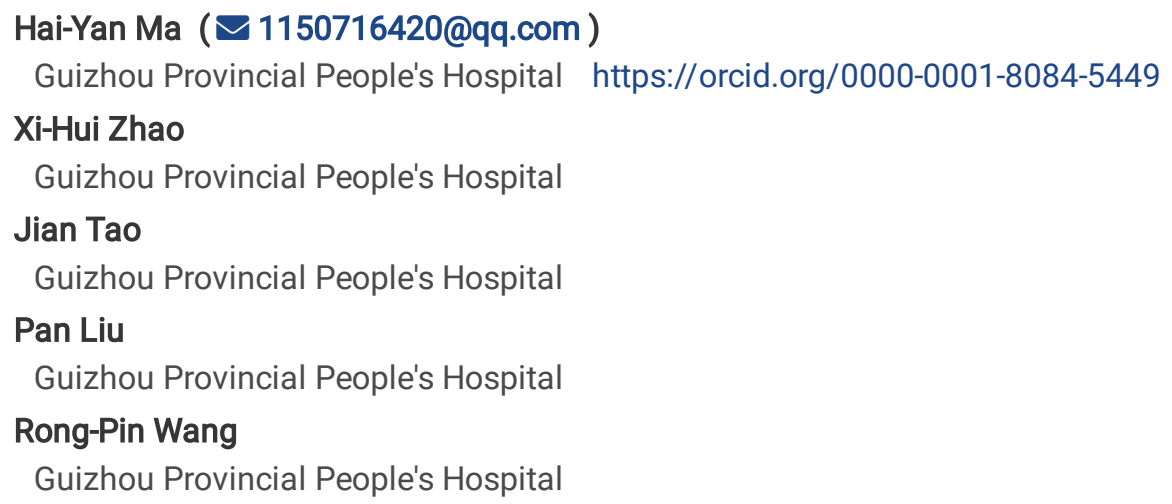

\section{Research Article}

Keywords: Cardiac magnetic resonance feature tracking, Non-ischemic dilated cardiomyopathy, Ventricular arrhythmias, Reproducibility of intra-class correlation coefficient

Posted Date: July 12th, 2021

DOI: https://doi.org/10.21203/rs.3.rs-444134/v1

License: (c) (i) This work is licensed under a Creative Commons Attribution 4.0 International License. Read Full License 


\section{Abstract}

This study investigated the feasibility of using cardiovascular magnetic resonance feature tracking (CMR-FT) for analysis of left ventricular (LV) strain and strain rate in patients with non-ischemic dilated cardiomyopathy (NIDCM) combined with ventricular arrhythmias (VAs). And evaluated the correlation between the LV global strain and left ventricular ejection (LVEF). We performed a retrospective study in a cohort of 34 consecutive patients with NIDCM combined with VAs who underwent CMR examination in our hospital between January 2016 and December 2019. Global and segmental peak values of LV longitudinal, circumferential, radial strain, and systolic strain rate were analyzed. Pearson analysis was calculated to assess the correlation of LV global deformation and LVEF as well as the correlation of between LV global deformation. Compared with the healthy controls, the global peak radial strain (GPRS), global peak circumferential strain (GPCS), and global peak longitudinal strain (GPLS) were significantly reduced in patients with NIDCM combined with VAs $(P<0.001$, respectively). Additionally, Pearson analysis showed GPCS negatively correlated with LVEF $(r=-0.946, P<0.001)$, GPLS negatively correlated with LVEF $(r=-0.860, P<0.001)$, and GPRS positively correlated with LVEF $(r=0.920, P$ $<0.001)$. CMR-FT is a feasible and promising technique for assessing LV myocardial deformation of patients with NIDCM combined with VAs. And, GPCS was better negatively correlated with LVEF and higher reproducibility of intra-class correlation coefficient (ICC), which can help to guide clinical treatment and have great implication on clinical decision.

\section{Introduction}

Non-ischemic dilated cardiomyopathy (NIDCM) is a myocardial disorder characterized by the presence of left ventricular (LV) or biventricular dilatation and systolic dysfunction in the absence of coronary artery disease or abnormal loading conditions proportionate to the degree of LV impairment[1-3]. In the process development of NIDCM, it may or may not develop overt heart failure (HF). Although modern management of HF increased the survival rates of NIDCM and has resulted in long periods of clinical stability, the 5-year mortality rate remains as high as $20 \%$ [4]. Moreover, in clinical practice, patients with dilated cardiomyopathy are prone to arrhythmias, and the cause of mortality in these patients is either end-organ dysfunction due to pump failure or arrhythmiarelated death [5]. Several studies that analyzed the ventricular arrhythmias (VAs) substrate found that myocardial fibrosis plays an important role in the genesis of VAs in patients with NIDCM [6-8]. Therefore, patients with NIDCM combined VAs should be continuously re-assessed, particularly in the presence of abrupt worsening of left ventricle (LV) function or an increased ventricular arrhythmic burden.

At present, novel cardiovascular magnetic resonance (CMR) techniques are rapidly emerging as useful and unique tools for a comprehensive cardiac evaluation of dilated cardiomyopathy, including evaluation of chamber size quantification, ventricular function and mass, myocardial wall thicknesses, segmental function, myocardial fibrosis, myocardial edema, and tissue characterization [9-11]. Myocardial strain allows quantitative measurement of global but also regional myocardial function and deformation offering additional information beyond ejection fraction[12,13]. Moreover, CMR-FT has high spatial, temporal resolution and nearly incompressible biventricular model, which is more accurate than speckle-tracking echocardiography [14-16].

However, the feasibility of CMR-based feature tracking strain analysis in patients with NIDCM combined with VAs has not been investigated. Therefore, the purpose of the current study was to observe the left ventricular myocardial strain for patients with NIDCM combined with VAs and study the correlation between the LV global strain values and LVEF, and relationship between pair-wise LV global strain values.

\section{Methods}

\section{Patient population}

We performed a retrospective study in a cohort of 96 consecutive suspected patients with NIDCM in our hospital between January 2016 and December 2019. Inclusion criteria were: (1) the definition and diagnosis of patients with NIDCM was made according to the CMR current recommendations [17], (2) all patients diagnosed with NIDCM underwent 24-hour Holter electrocardiography examinations, (3) all NIDCM patients with VAs which CMR-FT analysis could be performed were included. Exclusion criteria were: (1) coronary artery disease with $>50 \%$ stenosis on elective angiography, (2) NIDCM patients without ventricular arrhythmias based on 24-hour Holter electrocardiography examinations, (3) NIDCM patients with ventricular arrhythmias had performed radiofrequency ablation, (4) systemic disease, (5) hypertensive heart disease, (6) valvular disease, (7) congenital heart disease, (8) combined myocardial infarction, and (9) inappropriate image. 


\section{Control group}

As a control, 25 healthy volunteers who met the following inclusion criteria were recruited from our hospital: (i) no symptoms or prior history of cardiovascular disease; (ii) no prior diagnosis of hyperlipidaemia, hypertension or diabetes mellitus; (iii) normal physical examination and electrocardiogram; (iv) no contraindications to CMR. (v)CMR-FT analysis could be performed in all subjects.

This study was approved by the Ethics Committee of Guizhou Provincial People's Hospital (No. 2020-384) and it complies with the ethical principles of the Declaration of Helsinki (2000 EDITION). All enrolled healthy volunteers provided written informed consent prior to investigation. However, written informed consent for patients with NIDCM were waived by the Ethics Committee of Guizhou Provincial People's Hospital because this study on NIDCM patients was retrospective.

\section{Twenty-four-hour Holter electrocardiography (ECG) monitoring}

Three-channel 24-hour Holter ECG recordings were obtained in all patients in the absence of class I or class III antiarrhythmic drugs. The Holter recordings were analyzed by computer (Shenzhen Bosheng, China) with manual overread performed by an experienced doctor. All Holter readouts were confirmed by the independent senior observers blinded to clinical data.

\section{Cardiac magnetic resonance (CMR) imaging}

CMR imaging was performed using a 1.5-Tesla (Aera, Siemens, Germany), 1.5-Tesla (Verio, Siemens, Germany) and a standardized imaging protocol [18]. Balanced steady-state free precession sequence (SSFP)sequences with breath-hold were performed to obtain cine CMR images, comprising a stack of contiguous parallel short-axis slices covering the entire LV from base to apex and three LV long-axis slice (2-, 3-, and 4-chamber views) images. Cine images in the short axis (SA) planes were acquired at 1.5 Tesla using a balanced, SSFP sequence (repetition time/echo time/flip angle [TR/TE/FA] 2.4/1.2/50-75 degrees, matrix192×256,

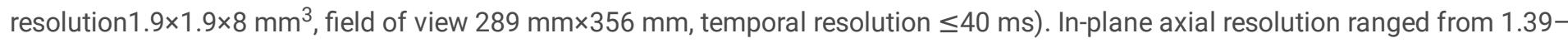
$1.5 \mathrm{~mm}$ per dimension. Delayed enhancement images were acquired at end-diastole during breath-holding using a segmented inversion-recovery gradient-echo turbo fast low angle shot sequence obtained 8-10min after injection of $0.2 \mathrm{mmol} / \mathrm{kilogram}$ of gadopentetate dimeglumine (Magnevist, Bayer) contrast medium. 18-channel phased-array chest coil was used for all data acquisition.

\section{CMR feature-tracking analysis}

CMR-FT analysis was performed with dedicated software (CVI42 v5.11, Circle Cardiovascular Imaging, Calgary, Canada). SAX and LAX cine images were uploaded into the software which reconstructed a 3D model and derived peak radial, circumferential and longitudinal strain [19]. CMR-FT post-processing software was used to analyze the LV strain, short-axis view, and 2- and 4-ch views of the ventricle, which were divided according to the American Heart Association (AHA) 17-segment model [20]. The apical cap (segment 17) was not considered for analysis. Global and regional LV strain and systolic strain rate measurements were analyzed and feature tracking allows quantification of global and regional longitudinal, circumferential and radial strain and strain rates. The quality of tracking was visually verified and manually adjusted if necessary. Inter-observer reproducibility was measured using a randomly selected sample of 20 cases by two independent observers.

In addition, LV end-diastolic volume (LVEDV), end-systolic volume (LVESV), stroke volume (LVSV), ejection fraction (LVEF) and LV mass were measured via standardized protocols using semi-automated commercial software (CVI42) [18,21]. And, the late gadolinium enhancement (LGE) mass and LV LGE percent (defined as the LGE mass divided by normal myocardial mass) were also quantitatively analyzed by commercially available software (CVI42). The LGE was defined by using a signal intensity threshold level of 5 standard deviations (SDs) above a remote myocardial region that was used as a reference on the same section.

\section{Statistical analysis}

Statistical analysis was performed with commercially available statistical software (IBM SPSS Statistics 20.0, Chicago, Illinois). Qualitative or quantitative variables were expressed as mean \pm standard deviation (SD), percentages, or median (interquartile range) as appropriate. All data were controlled for a normal distribution by the Kolmogorov-Smirnov test before analyzing the differences between two groups data. For continuous variables, differences between two groups were compared using Unpaired Students' t-test or 
Mann-Whitney $\mathrm{U}$ test. The distribution of qualitative variables was analyzed by chi-square test or Fisher's exact test. The association between the LVEF and LV strain parameters was assessed using Pearson correlation in all patients. And, the correlation between the LV strain parameters was observed using Pearson correlation in all patients. A two-tailed $\mathrm{P}<0.05$ was considered statistically significant. The intraobserver and interobserver variability for LV strain values were studied in a group of 20 randomly selected subjects by one observer, repeated twice, and by two observers who were unaware of each other's measurements.

\section{Results}

\section{Baseline characteristics of the study participants}

Of the 96 consecutive suspected patients with NIDCM initially enrolled in the study, 62 subjects were excluded according to inclusion and exclusion criteria. Thus, in total, 34 patients diagnosed with NIDCM combined with VAs (67\% were males, mean age was $46 \pm 14$ years) and 25 healthy controls were finally included. And, CMR-FT analysis could be performed in all subjects. No patients had LGE with an ischemic pattern.

There was no statistical difference between the NIDCM combined VAs group and the control group in terms of sex and body surface area(BSA) $(P>0.05$, for each), whereas age had difference between NIDCM patients and controls $(P<0.05)$. Baseline characteristics in the NIDCM combined VAs group and the control group are presented in Table 1. 
Table 1

Baseline characteristics of patients with NIDCM and controls

\begin{tabular}{|c|c|c|c|c|}
\hline Variable & $\operatorname{DCM}(n=34)$ & Controls $(n=25)$ & $\mathrm{t} / / \chi^{2} / \mathrm{z}$ & $P$ value \\
\hline Age (years) & $46 \pm 14$ & $29 \pm 6$ & -5.57 & $<0.001$ \\
\hline Sex (male) & $23(67 \%)$ & $15(60 \%)$ & 1.23 & 0.436 \\
\hline $\operatorname{BSA}\left(\mathrm{m}^{2}\right)$ & $1.76(1.49,1.83)$ & $1.70(1.59,1.80)$ & -0.26 & 0.793 \\
\hline Clinical Symptom & Palpitation, chest distress & - & - & - \\
\hline \multicolumn{5}{|l|}{ Holter $\mathrm{n}(\%)$} \\
\hline PVCs & $18(53 \%)$ & - & - & - \\
\hline NSVT & $15(44 \%)$ & - & - & - \\
\hline SVT & $1(3 \%)$ & - & - & - \\
\hline LVEF (\%) & $22.23 \pm 7.86$ & $63.21 \pm 5.13$ & 22.72 & $<0.001$ \\
\hline $\mathrm{EDV}(\mathrm{mL})$ & $287.45 \pm 85.26$ & $114.19 \pm 22.48$ & -9.89 & $<0.001$ \\
\hline $\mathrm{ESV}(\mathrm{mL})$ & $223.63 \pm 85.01$ & $45.99 \pm 12.47$ & -10.34 & $<0.001$ \\
\hline $\mathrm{SV}(\mathrm{mL})$ & $50.37 \pm 15.35$ & $82.31 \pm 11.56$ & 8.73 & $<0.001$ \\
\hline $\mathrm{CO}(\mathrm{L} / \mathrm{min})$ & $3.62 \pm 0.87$ & $5.26 \pm 0.82$ & 7.32 & $<0.001$ \\
\hline $\mathrm{Cl}\left(\mathrm{L} / \mathrm{min} / \mathrm{m}^{2}\right)$ & $2.70(1.98,3.49)$ & $4.27(3.38,4.42)$ & -4.65 & $<0.001$ \\
\hline $\operatorname{LVEDVI}\left(\mathrm{mL} / \mathrm{m}^{2}\right)$ & $172.39 \pm 45.10$ & $69.84 \pm 15.33$ & -10.89 & $<0.001$ \\
\hline $\operatorname{LVESVI}\left(\mathrm{mL} / \mathrm{m}^{2}\right)$ & $132.82 \pm 46.03$ & $27.59 \pm 7.44$ & -11.29 & $<0.001$ \\
\hline $\mathrm{SVI}\left(\mathrm{mL} / \mathrm{m}^{2}\right)$ & $39.62 \pm 16.07$ & $42.09 \pm 10.39$ & 0.674 & 0.503 \\
\hline LGE positive, n (\%) & $29(85 \%)$ & - & - & - \\
\hline LGE mass $(\mathrm{g})$ & $12.16 \pm 3.87$ & - & - & - \\
\hline LGE percent (\%) & $11.73 \pm 3.42$ & - & - & - \\
\hline \multicolumn{5}{|c|}{$\begin{array}{l}\text { Results are reported as mean } \pm \text { SD, percentages, or median (interquartile range) as appropriate. BSA, body surface area; LVEF, lef } \\
\text { ventricular ejection; EDV, end-diastolic volume; ESV, end-systolic volume; SV, Stroke volume; CO, cardiac output; CI, Cardiac index; } \\
\text { LVEDVI, indexed-left ventricular end diastolic volume; LVESVI, indexed-left ventricular end systolic volume; SVI, indexed-stroke } \\
\text { volume; PVCs, Premature ventricular contractions; NSVT, nonsustained ventricular tachycardia; SVT, sustained ventricular } \\
\text { tachycardia; LGE, the late gadolinium enhancement }\end{array}$} \\
\hline
\end{tabular}

\section{Cardiac Function Parameters}

LGE was present in 29 (85\%) of NIDCM patients with a mean global LGE mass of $12.16 \pm 3.87$. The LVEF, LVEDV, LVESV, SV, CO, Cl, LVEDVI and LVEDVI were significantly lower in the patients with NIDCM combined VAs than in the controls $(P<0.001$ for all), whereas SVI had no differences between NIDCM patients and controls $(P>0.05)$. Patient cardiac function parameters data is summarized in Table 1.

\section{4-hour Holter Findings}

A total of 34 patients with NIDCM combined VAs were diagnosed after underwent 24 Holter electrocardiogram examinations. The 24hour Holter documented 15 patients (44\%) with nonsustained ventricular tachycardia, 11 patients (32\%) with frequent premature ventricular beats (ie, $>1,000$ over 24 hours of Holter monitoring), 7 patients $(21 \%)$ with frequent premature ventricular beats (ie, $<1,000$ over 24 hours of Holter monitoring), and 1 patient (3\%)with sustained ventricular tachycardia. 


\section{CMR Global And Segmental Strain Analysis}

At the LV global strains level, patients with NIDCM combined VAs demonstrated significantly impaired LV GPRS, GPCS, GPLS GPRSR, GPCSR and GPLSR compared to healthy controls $(P<0.001$ for all) in Table 2. For segmental strain level, we found that patients with NIDCM with VAs had significantly lower PLS, PLSR, PCS, PCSR, PRS, and PRSR values at most segments to different degrees, compared with the control group $(\mathrm{P}<0.001$ for each); whereas the only exception was the PRS at the middle anterior lateral segment, PLS at the apical anterior lateral, apical septal and middle anterior lateral segments, and PRSR at the middle septal inferior and middle anterior segments, where no significant difference could be identified $(P>0.05)$ (Table 3 , and 4). And, a representative example of the derivation of strain in patient with NIDCM combined VAs in Fig. 1.

Table 2

Global strain of NIDCM combined with VAs and controls

\begin{tabular}{|lllll|}
\hline Variable & NIDCM$(\mathbf{n}=\mathbf{3 4})$ & Controls $(\mathbf{n}=\mathbf{2 5})$ & $\mathrm{t} / \mathbf{Z}$ & P value \\
\hline GPRS & $8.21 \pm 2.78$ & $41.69 \pm 5.38$ & 31.16 & $<0.001$ \\
\hline GPCS & $-5.99 \pm 3.73$ & $-21.75 \pm 2.54$ & -18.22 & $<0.001$ \\
\hline GPLS & $-7.06 \pm 2.25$ & $-15.54 \pm 1.23$ & -17.04 & $<0.001$ \\
\hline GPRSR & $0.69 \pm 0.39$ & $1.91 \pm 0.48$ & 10.80 & $<0.001$ \\
\hline GPCSR & $-0.37(-0.54,-0.17)$ & $-1.37(-1.52,-1.28)$ & -6.48 & $<0.001$ \\
\hline GPLSR & $-0.23 \pm 0.18$ & $-0.74 \pm 0.15$ & -11.39 & $<0.001$ \\
\hline
\end{tabular}

Results are reported as mean \pm SD or median (interquartile range) as appropriate. NIDCM, nonischemic dilated cardiomyopathy; GPRS, global peak radial strain; GPRSR, global peak systolic radial strain rate; GPCS, global peak circumferential strain; GPRSR, global peak systolic circumferential strain rate; GPLS, global peak longitudinal strain; GPLSR, global peak systolic longitudinal strain rate 
Table 3

Segmental values of strain in patients with NIDCM combined with VAs and controls

\begin{tabular}{|c|c|c|c|c|c|c|c|c|c|}
\hline \multirow{2}{*}{$\begin{array}{l}\text { Variable } \\
\text { segment }\end{array}$} & \multicolumn{2}{|l|}{ PRS } & \multirow[t]{2}{*}{$\mathbf{P}$} & \multicolumn{2}{|l|}{ PCS } & \multirow[t]{2}{*}{$P$} & \multicolumn{2}{|l|}{ PLS } & \multirow[t]{2}{*}{$\mathbf{P}$} \\
\hline & Controls & NIDCM & & Controls & NIDCM & & Controls & NIDCM & \\
\hline \multicolumn{10}{|l|}{ Basal } \\
\hline \multirow[t]{2}{*}{ anterior } & $47.75 \pm 6.09$ & $5.79 \pm 3.40$ & $<0.001$ & $\begin{array}{l}-16.26 \pm \\
2.35\end{array}$ & $\begin{array}{l}-5.73 \pm \\
2.55\end{array}$ & $\begin{array}{l}< \\
0.001\end{array}$ & $\begin{array}{l}-17.34(- \\
18.91\end{array}$ & $\begin{array}{l}-7.40(- \\
8.36\end{array}$ & $\begin{array}{l}< \\
0.001\end{array}$ \\
\hline & & & & & & & $-16.42)$ & $-6.28)$ & \\
\hline \multirow[t]{2}{*}{$\begin{array}{l}\text { Anterior } \\
\text { septal }\end{array}$} & $56.98 \pm 6.86$ & $9.05 \pm 4.63$ & $<0.001$ & $\begin{array}{l}-19.78(- \\
22.06,\end{array}$ & $\begin{array}{l}-6.14(- \\
7.23\end{array}$ & $\begin{array}{l}< \\
0.001\end{array}$ & $\begin{array}{l}-17.28(- \\
18.67,\end{array}$ & $\begin{array}{l}-6.37(- \\
7.16\end{array}$ & $\begin{array}{l}< \\
0.001\end{array}$ \\
\hline & & & & $-17.83)$ & $-4.99)$ & & $-14.53)$ & $-5.02)$ & \\
\hline \multirow[t]{2}{*}{$\begin{array}{l}\text { interior } \\
\text { septal }\end{array}$} & $50.34(46.68,55.13)$ & $8.27(7.46,11.32)$ & $<.001$ & $\begin{array}{l}-16.38 \pm \\
4.44\end{array}$ & $\begin{array}{l}-7.07 \pm \\
3.51\end{array}$ & $<.001$ & $\begin{array}{l}-9.83(- \\
\text { 14.37, }\end{array}$ & $\begin{array}{l}-5.22(- \\
6.46\end{array}$ & $<.001$ \\
\hline & & & & & & & $-7.61)$ & $-4.19)$ & \\
\hline interior & $43.77 \pm 4.94$ & $10.84 \pm 3.35$ & $<.001$ & $\begin{array}{l}-9.80 \pm \\
4.99\end{array}$ & $\begin{array}{l}-5.75 \pm \\
2.93\end{array}$ & $\begin{array}{l}< \\
0.001\end{array}$ & $\begin{array}{l}-19.10 \pm \\
4.33\end{array}$ & $\begin{array}{l}-5.90 \pm \\
2.11\end{array}$ & $<0.001$ \\
\hline \multirow[t]{2}{*}{$\begin{array}{l}\text { inferior } \\
\text { lateral }\end{array}$} & $50.14(46.22,58.36)$ & $5.28(4.13,8.23)$ & $<.001$ & $\begin{array}{l}-6.24(- \\
9.99\end{array}$ & $\begin{array}{l}-5.15(- \\
6.17\end{array}$ & 0.002 & $\begin{array}{l}-16.11(- \\
18.78,\end{array}$ & $\begin{array}{l}-6.97(- \\
8.11\end{array}$ & $<0.001$ \\
\hline & & & & $-5.26)$ & $-4.13)$ & & $-14.33)$ & $-5.29)$ & \\
\hline \multirow[t]{2}{*}{$\begin{array}{l}\text { anterior } \\
\text { lateral }\end{array}$} & $57.25(54.76,63.17)$ & $5.33(3.61,8.31)$ & $<.001$ & $\begin{array}{l}-10.22(- \\
15.45\end{array}$ & $\begin{array}{l}-4.34(- \\
6.09\end{array}$ & $\dot{0} 0001$ & $\begin{array}{l}-15.53(- \\
17.46\end{array}$ & $\begin{array}{l}-7.91(- \\
8.81\end{array}$ & $\begin{array}{l}< \\
0.001\end{array}$ \\
\hline & & & & $-7.91)$ & $-4.14)$ & & $-14.39)$ & $-4.53)$ & \\
\hline \multicolumn{10}{|l|}{ Middle } \\
\hline \multirow[t]{2}{*}{ anterior } & $47.32(41.89,52.18)$ & $6.12(4.37,7.19)$ & $<.001$ & $\begin{array}{l}-18.75(- \\
20.17\end{array}$ & $\begin{array}{l}-6.61(- \\
8.03\end{array}$ & $<.001$ & $\begin{array}{l}-4.55(- \\
8.76,\end{array}$ & $\begin{array}{l}-2.12(- \\
3.21\end{array}$ & $\begin{array}{l}< \\
0.001\end{array}$ \\
\hline & & & & $-15.56)$ & $-5.41)$ & & $-3.36)$ & $-1.05)$ & \\
\hline \multirow[t]{2}{*}{$\begin{array}{l}\text { anterior } \\
\text { septal }\end{array}$} & $42.47(39.36,46.73)$ & $5.15(3.99,9.37)$ & $<.001$ & $\begin{array}{l}-23.57(- \\
26.27\end{array}$ & $\begin{array}{l}-6.48(- \\
9.08\end{array}$ & $<.001$ & $\begin{array}{l}-6.31(- \\
8.29\end{array}$ & $\begin{array}{l}-5.22(- \\
6.33\end{array}$ & 0.025 \\
\hline & & & & $-21.16)$ & $-4.98)$ & & $-4.17)$ & $-3.09)$ & \\
\hline inferoseptal & $38.77 \pm 5.52$ & $9.19 \pm 3.27$ & $<0.001$ & $\begin{array}{l}-19.43 \pm \\
2.13\end{array}$ & $\begin{array}{l}-6.80 \pm \\
1.94\end{array}$ & $<.001$ & $\begin{array}{l}-9.05 \pm \\
4.52\end{array}$ & $\begin{array}{l}-3.26 \pm \\
1.76\end{array}$ & $\begin{array}{l}< \\
0.001\end{array}$ \\
\hline \multirow[t]{2}{*}{ inferior } & $42.79 \pm 4.89$ & $7.39 \pm 2.88$ & $<0.001$ & $\begin{array}{l}-15.92(- \\
17.25\end{array}$ & $\begin{array}{l}-6.76(- \\
8.10\end{array}$ & $<0.001$ & $\begin{array}{l}-12.68(- \\
16.53,\end{array}$ & $\begin{array}{l}-4.85(- \\
6.33\end{array}$ & $<0.001$ \\
\hline & & & & $-13.78)$ & $-5.72)$ & & $-8.64)$ & $-3.27)$ & \\
\hline \multirow[t]{2}{*}{$\begin{array}{l}\text { inferior } \\
\text { lateral }\end{array}$} & $51.22(47.18,56.77)$ & $7.08(5.01,8.83)$ & $<.001$ & $\begin{array}{l}-15.27(- \\
18.88,\end{array}$ & $\begin{array}{l}-5.79(- \\
7.15\end{array}$ & $\begin{array}{l}<.001 \\
0.00\end{array}$ & $\begin{array}{l}-16.55(- \\
18.13\end{array}$ & $\begin{array}{l}-5.54(- \\
6.62,\end{array}$ & $\begin{array}{l}<.001 \\
0.001\end{array}$ \\
\hline & & & & $-10.90)$ & $-4.66)$ & & $-13.80)$ & $-3.69)$ & \\
\hline \multirow[t]{2}{*}{$\begin{array}{l}\text { anterior } \\
\text { lateral }\end{array}$} & $49.69 \pm 6.45$ & $13.76 \pm 3.38$ & $<0.001$ & $\begin{array}{l}-12.34(- \\
14.23\end{array}$ & $\begin{array}{l}-6.14(- \\
7.34\end{array}$ & $\begin{array}{l}< \\
0.001\end{array}$ & $\begin{array}{l}-6.53(- \\
8.02\end{array}$ & $\begin{array}{l}-6.14(- \\
7.40,\end{array}$ & 0.362 \\
\hline & & & & $-8.29)$ & $-5.14)$ & & $-5.03)$ & $-4.92)$ & \\
\hline \multicolumn{10}{|l|}{ Apical } \\
\hline \multirow[t]{2}{*}{ anterior } & $49.77 \pm 10.12$ & $14.33 \pm 4.61$ & $<.001$ & $\begin{array}{l}-17.24(- \\
19.45\end{array}$ & $\begin{array}{l}-5.02(- \\
6.08\end{array}$ & $<.001$ & $\begin{array}{l}-3.07(- \\
6.33\end{array}$ & $\begin{array}{l}-3.22(- \\
4.51\end{array}$ & 0.334 \\
\hline & & & & $-14.71)$ & $-4.09)$ & & $-1.51)$ & $-1.14)$ & \\
\hline
\end{tabular}

PRS, segmental peak radial strain; PCS, segmental peak circumferential strain; PLS, segmental peak longitudinal strain 


\begin{tabular}{|c|c|c|c|c|c|c|c|c|c|}
\hline \multirow{2}{*}{$\begin{array}{l}\text { Variable } \\
\text { segment }\end{array}$} & \multicolumn{2}{|l|}{ PRS } & \multirow[t]{2}{*}{$\mathbf{P}$} & \multicolumn{2}{|l|}{ PCS } & \multirow[t]{2}{*}{$P$} & \multicolumn{2}{|l|}{ PLS } & \multirow[t]{2}{*}{$P$} \\
\hline & Controls & NIDCM & & Controls & NIDCM & & Controls & NIDCM & \\
\hline \multirow[t]{2}{*}{ septal } & $52.27 \pm 6.59$ & $12.68 \pm 4.62$ & $\begin{array}{l}<.001 \\
0.00\end{array}$ & $\begin{array}{l}-18.09(- \\
22.35\end{array}$ & $\begin{array}{l}-6.56(- \\
7.30\end{array}$ & $\dot{0} .001$ & $\begin{array}{l}-4.33(- \\
6.57,\end{array}$ & $\begin{array}{l}-3.95(- \\
4.67,\end{array}$ & 0.096 \\
\hline & & & & $-15.64)$ & $-5.78)$ & & $-3.43)$ & $-1.16)$ & \\
\hline \multirow[t]{2}{*}{ inferior } & $10.29 \pm 3.63$ & $9.37 \pm 4.85$ & 0.153 & $\begin{array}{l}-20.11(- \\
24.81\end{array}$ & $\begin{array}{l}-8.39(- \\
9.59,\end{array}$ & $\begin{array}{l}< \\
0.001\end{array}$ & $\begin{array}{l}-5.41(- \\
7.39,\end{array}$ & $\begin{array}{l}-2.04(- \\
4.21,\end{array}$ & $\hat{0.001}$ \\
\hline & & & & $-18.22)$ & $-6.69)$ & & $-3.35)$ & $-0.94)$ & \\
\hline \multirow[t]{2}{*}{ lateral } & $14.99 \pm 5.85$ & $13.42 \pm 4.89$ & 0.524 & $\begin{array}{l}-25.84(- \\
27.14\end{array}$ & $\begin{array}{l}-8.24(- \\
9.18\end{array}$ & $\begin{array}{l}< \\
0.001\end{array}$ & $\begin{array}{l}-6.69 \pm \\
2.49\end{array}$ & $\begin{array}{l}-3.30 \pm \\
2.59\end{array}$ & $\begin{array}{l}<.001 \\
0.00\end{array}$ \\
\hline & & & & $-22.34)$ & $-7.28)$ & & & & \\
\hline
\end{tabular}

PRS, segmental peak radial strain; PCS, segmental peak circumferential strain; PLS, segmental peak longitudinal strain 
Table 4

Segmental values of strain rate in patients with NIDCM combined VAs and controls

\begin{tabular}{|c|c|c|c|c|c|c|c|c|c|}
\hline \multirow{2}{*}{$\begin{array}{l}\text { Variable } \\
\text { segment }\end{array}$} & \multicolumn{2}{|l|}{ PRSR } & \multirow[t]{2}{*}{$\mathrm{P}$} & \multicolumn{2}{|l|}{ PCSR } & \multirow[t]{2}{*}{$P$} & \multicolumn{2}{|l|}{ PLSR } & \multirow[t]{2}{*}{$\mathbf{P}$} \\
\hline & Controls & NIDCM & & Controls & NIDCM & & Controls & NIDCM & \\
\hline \multicolumn{10}{|l|}{ Basal } \\
\hline \multirow[t]{2}{*}{ anterior } & $2.47(2.12,3.12)$ & $1.16(0.82,1.45)$ & $\begin{array}{l}< \\
0.001\end{array}$ & $\begin{array}{l}-1.90(- \\
2.36\end{array}$ & $\begin{array}{l}-0.64(- \\
1.10\end{array}$ & $\begin{array}{l}< \\
0.001\end{array}$ & $\begin{array}{l}-1.34(- \\
1.69,\end{array}$ & $\begin{array}{l}-0.97(- \\
1.17\end{array}$ & 0.003 \\
\hline & & & & $-1.25)$ & $-0.30)$ & & $-0.93)$ & $-0.59)$ & \\
\hline $\begin{array}{l}\text { Anterior } \\
\text { septal }\end{array}$ & $3.39 \pm 0.41$ & $1.26 \pm 0.92$ & $\begin{array}{l}< \\
0.001\end{array}$ & $\begin{array}{l}-1.75 \pm \\
0.59\end{array}$ & $\begin{array}{l}-0.68 \pm \\
0.54\end{array}$ & $\begin{array}{l}< \\
0.001\end{array}$ & $\begin{array}{l}-1.09 \pm \\
0.33\end{array}$ & $\begin{array}{l}-0.76 \pm \\
0.49\end{array}$ & 0.006 \\
\hline \multirow[t]{2}{*}{$\begin{array}{l}\text { interior } \\
\text { septal }\end{array}$} & $2.07(1.47,2.41)$ & $1.26(0.86,1.75)$ & $\begin{array}{l}< \\
0.001\end{array}$ & $\begin{array}{l}-1.23(- \\
1.71\end{array}$ & $\begin{array}{l}-0.55(- \\
0.84\end{array}$ & $\begin{array}{l}< \\
0.001\end{array}$ & $\begin{array}{l}-1.24 \pm \\
0.58\end{array}$ & $\begin{array}{l}-0.86 \pm \\
0.45\end{array}$ & 0.008 \\
\hline & & & & $-0.90)$ & $-0.37)$ & & & & \\
\hline interior & $2.15 \pm 0.62$ & $1.51 \pm 0.82$ & $<.001$ & $\begin{array}{l}-1.19 \pm \\
0.68\end{array}$ & $\begin{array}{l}-0.66 \pm \\
0.51\end{array}$ & $\begin{array}{l}< \\
0.001\end{array}$ & $\begin{array}{l}-1.10 \pm \\
0.53\end{array}$ & $\begin{array}{l}-0.74 \pm \\
0.57\end{array}$ & 0.017 \\
\hline \multirow[t]{2}{*}{$\begin{array}{l}\text { inferior } \\
\text { lateral }\end{array}$} & $3.36 \pm 1.06$ & $1.67 \pm 0.71$ & $\begin{array}{l}< \\
0.001\end{array}$ & $\begin{array}{l}-1.66(- \\
1.84\end{array}$ & $\begin{array}{l}-0.56(- \\
0.96\end{array}$ & $\begin{array}{l}<.001 \\
0.001\end{array}$ & $\begin{array}{l}-1.17 \pm \\
0.34\end{array}$ & $\begin{array}{l}-0.77 \pm \\
0.54\end{array}$ & 0.002 \\
\hline & & & & $-1.25)$ & $-0.36)$ & & & & \\
\hline $\begin{array}{l}\text { anterior } \\
\text { lateral }\end{array}$ & $3.91 \pm 1.14$ & $1.81 \pm 1.01$ & $\begin{array}{l}< \\
0.001\end{array}$ & $\begin{array}{l}-1.65 \pm \\
0.42\end{array}$ & $\begin{array}{l}-0.80 \pm \\
0.50\end{array}$ & $\begin{array}{l}< \\
0.001\end{array}$ & $\begin{array}{l}-1.20 \pm \\
0.49\end{array}$ & $\begin{array}{l}-0.80 \pm \\
0.50\end{array}$ & 0.003 \\
\hline \multicolumn{10}{|l|}{ Middle } \\
\hline \multirow[t]{2}{*}{ anterior } & $3.09 \pm 1.04$ & $1.98 \pm 1.14$ & 0.004 & $\begin{array}{l}-1.69 \pm \\
0.25\end{array}$ & $\begin{array}{l}-0.87 \pm \\
0.63\end{array}$ & $\begin{array}{l}< \\
0.001\end{array}$ & $\begin{array}{l}-1.08(- \\
1.33\end{array}$ & $\begin{array}{l}-0.88(- \\
1.17,\end{array}$ & 0.034 \\
\hline & & & & & & & $-0.81)$ & $-0.51)$ & \\
\hline \multirow[t]{2}{*}{$\begin{array}{l}\text { anterior } \\
\text { septal }\end{array}$} & $2.15(1.79,3.04)$ & $1.16(0.64,1.60)$ & $\begin{array}{l}< \\
0.001\end{array}$ & $\begin{array}{l}-1.36 \pm \\
0.55\end{array}$ & $\begin{array}{l}-0.63 \pm \\
0.71\end{array}$ & $\begin{array}{l}< \\
0.001\end{array}$ & $\begin{array}{l}-1.06(- \\
1.29,\end{array}$ & $\begin{array}{l}-0.64(- \\
1.02,\end{array}$ & $<.001$ \\
\hline & & & & & & & $-0.90)$ & $-0.28)$ & \\
\hline inferoseptal & $1.75(1.36,2.07)$ & $0.76(0.51,1.13)$ & $<0.001$ & $\begin{array}{l}-1.50 \pm \\
0.34\end{array}$ & $\begin{array}{l}-0.79 \pm \\
0.53\end{array}$ & $\begin{array}{l}< \\
0.001\end{array}$ & $\begin{array}{l}-1.15 \pm \\
0.34\end{array}$ & $\begin{array}{l}-0.72 \pm \\
0.39\end{array}$ & $\begin{array}{l}< \\
0.001\end{array}$ \\
\hline inferior & $1.82 \pm 0.76$ & $0.91 \pm 0.63$ & $<.001$ & $\begin{array}{l}-1.37 \pm \\
0.42\end{array}$ & $\begin{array}{l}-0.85 \pm \\
0.48\end{array}$ & $\begin{array}{l}<.001 \\
0.001\end{array}$ & $\begin{array}{l}-1.16 \pm \\
0.36\end{array}$ & $\begin{array}{l}-0.77 \pm \\
0.50\end{array}$ & 0.002 \\
\hline $\begin{array}{l}\text { inferior } \\
\text { lateral }\end{array}$ & $2.56(1.98,3.28)$ & $0.99(0.57,1.20)$ & $<0.001$ & $\begin{array}{l}-1.82 \pm \\
0.43\end{array}$ & $\begin{array}{l}-0.88 \pm \\
0.67\end{array}$ & $\begin{array}{l}< \\
0.001\end{array}$ & $\begin{array}{l}-1.46 \pm \\
0.57\end{array}$ & $\begin{array}{l}-0.92 \pm \\
0.35\end{array}$ & $\begin{array}{l}< \\
0.001\end{array}$ \\
\hline \multirow[t]{2}{*}{$\begin{array}{l}\text { anterior } \\
\text { lateral }\end{array}$} & $2.91 \pm 0.77$ & $1.30 \pm 0.80$ & $<.001$ & $\begin{array}{l}-1.62 \pm \\
0.43\end{array}$ & $\begin{array}{l}-0.79 \pm \\
0.58\end{array}$ & $<0.001$ & $\begin{array}{l}-1.06(- \\
1.25\end{array}$ & $-0.58(-0.95$ & 0.004 \\
\hline & & & & & & & & $-0.34)$ & \\
\hline \multicolumn{10}{|l|}{ Apical } \\
\hline \multirow[t]{2}{*}{ anterior } & $2.29 \pm 0.61$ & $1.08 \pm 0.49$ & $<0.001$ & $\begin{array}{l}-1.32(- \\
1.78,\end{array}$ & $\begin{array}{l}-0.92(- \\
1.25\end{array}$ & $\begin{array}{l}< \\
0.001\end{array}$ & $\begin{array}{l}-0.96 \pm \\
0.45\end{array}$ & $\begin{array}{l}-0.50 \pm \\
0.52\end{array}$ & 0.001 \\
\hline & & & & $-1.17)$ & $-0.46)$ & & & & \\
\hline septal & $2.14 \pm 0.34$ & $1.08 \pm 0.47$ & 0.001 & $\begin{array}{l}-1.34 \pm \\
0.30\end{array}$ & $\begin{array}{l}-0.78 \pm \\
0.37\end{array}$ & $\begin{array}{l}< \\
0.001\end{array}$ & $\begin{array}{l}-1.14 \pm \\
0.38\end{array}$ & $\begin{array}{l}-1.05 \pm \\
0.46\end{array}$ & 0.003 \\
\hline
\end{tabular}




\begin{tabular}{|c|c|c|c|c|c|c|c|c|c|}
\hline \multirow{2}{*}{$\begin{array}{l}\text { Variable } \\
\text { segment }\end{array}$} & \multicolumn{2}{|l|}{ PRSR } & \multirow[t]{2}{*}{$\mathbf{P}$} & \multicolumn{2}{|l|}{ PCSR } & \multirow[t]{2}{*}{$P$} & \multicolumn{2}{|l|}{ PLSR } & \multirow[t]{2}{*}{$\mathbf{P}$} \\
\hline & Controls & NIDCM & & Controls & NIDCM & & Controls & NIDCM & \\
\hline \multirow[t]{2}{*}{ inferior } & $3.89 \pm 1.05$ & $1.11 \pm 0.65$ & $\dot{0} .001$ & $\begin{array}{l}-1.51(- \\
1.77,\end{array}$ & $\begin{array}{l}-0.76(- \\
1.09\end{array}$ & $\begin{array}{l}<.001 \\
0.00\end{array}$ & $\begin{array}{l}-1.28 \pm \\
0.34\end{array}$ & $\begin{array}{l}-0.78 \pm \\
0.42\end{array}$ & $<.001$ \\
\hline & & & & $-1.39)$ & $-0.46)$ & & & & \\
\hline \multirow[t]{2}{*}{ lateral } & $3.23(2.62,4.26)$ & $1.20(0.77,1.68)$ & $\begin{array}{l}< \\
0.001\end{array}$ & $\begin{array}{l}-1.55(- \\
1.83\end{array}$ & $\begin{array}{l}-0.86(- \\
1.13\end{array}$ & $\begin{array}{l}<.001 \\
0.00\end{array}$ & $\begin{array}{l}-1.24 \pm \\
0.39\end{array}$ & $\begin{array}{l}-0.76 \pm \\
0.46\end{array}$ & $<.001$ \\
\hline & & & & $-1.23)$ & $-0.58)$ & & & & \\
\hline
\end{tabular}

\section{Correlation Between The Lv Global Strain Values And Lvef}

GPCS negatively correlated with LVEF $(r=-0.946, P<0.001)$, GPLS negatively correlated with LVEF $(r=-0.860, P<0.001)$, and GPRS positively correlated with LVEF $(r=0.920, P<0.001)$, as shown in Fig. 2A-C. And, GPCSR negatively correlated with LVEF $(r=-0.840, P<$ $0.001)$, GPLSR negatively correlated with LVEF $(r=-0.767, P<0.001)$, and GPRSR positively correlated with $\operatorname{LVEF}(r=0.742, P<0.001)$, as shown in Fig. 2D-F.

\section{Correlation Between With Pair-wise Comparison Lv Global Strain Values}

With pair-wise comparison, we identified both GPCS and GPLS were negative correlated with GPRS, while the GPCS and GPLS were positively correlated with each other (Fig. 3A-C (GPCS vs. GPRS: $r=-0.896, P<0.001$; GPRS vs. GPLS, $r=-0.906, P<0.001$; GPCS vs. GPLS, $r=0.834, P<0.001$ ). Additionally, we also proved a negative correlation between the GPCSR and GPRSR as well as between the GPRSR and GPLSR, while the GPCSR and GPLSR were positively correlated with each other (Fig. 3D-F (GPCSR vs. GPRSR: $r=-0.757$, $P<0.001$; GPRSR vs. GPLSR, $r=-0.804, P<0.001$; GPCSR vs. GPLSR, $r=0.818, P<0.001$ ).

\section{Intra-observer And Inter-observer Variability}

Intra-observer and inter-observer reproducibility analysis for LV global strains parameters was tested and presented in Tables 5.

Tables 5 Intra- and inter-observer reproducibility for left ventricle global strain

\begin{tabular}{|lllll|}
\hline \multirow{2}{*}{ Parameters } & \multicolumn{2}{l}{ Intra-observer agreement } & \multicolumn{2}{l|}{ Inter-observer agreement } \\
\cline { 2 - 5 } & ICC $[95 \% \mathrm{Cl}]$ & Mean difference \pm SD & ICC [95\%Cl] & Mean difference \pm SD \\
\hline GPRS $(\%)$ & $0.93(0.80-0.97)$ & $13.04 \pm 10.28$ & $0.90(0.89-0.96)$ & $1.43 \pm 1.32$ \\
\hline GPCS $(\%)$ & $0.96(0.89-0.98)$ & $4.67 \pm 5.03$ & $0.96(0.90-0.98)$ & $2.09 \pm 1.54$ \\
\hline GPLS (\%) & $0.92(0.78-0.97)$ & $10.67 \pm 8.36$ & $0.93(0.83-0.99)$ & $3.98 \pm 3.11$ \\
\hline GPRSR(1/s) & $0.89(0.75-0.95)$ & $4.82 \pm 5.02$ & $0.89(0.78-0.96)$ & $1.49 \pm 1.34$ \\
\hline GPCSR(1/s) & $0.95(0.91-0.99)$ & $6.40 \pm 4.79$ & $0.92(0.79-0.97)$ & $0.78 \pm 0.53$ \\
\hline GPLSR(1/s) & $0.93(0.80-0.97)$ & $8.33 \pm 11.23$ & $0.91(0.85-0.96)$ & $3.86 \pm 3.02$ \\
\hline $\begin{array}{l}\text { GPRS, global peak radial strain; GPRSR, global peak systolic radial strain rate; GPCS, global peak circumferential strain; GPRSR, } \\
\text { global peak systolic circumferential strain rate; GPLS, global peak longitudinal strain; GPLSR, global peak systolic longitudinal } \\
\text { strain rate }\end{array}$ & & & \\
\hline
\end{tabular}

\section{Discussion}


To the best of our knowledge, the present study is the first to assess the feasibility and performance in patients with NIDCM combined with VAs using CMR-FT methods. Firstly, we found that LV deformation analysis can differentiate patients NIDCM with VAs from controls using CMR-FT. Secondly, we proved that the LV global strain values had a linear correlation with LVEF. Moreover, we performed pair-wise comparison among the LV global strain values, which showed linear correlations among one another. These findings which to detect early deregulated LV function in patients with NIDCM with VAs have great implication on clinical decision, which might help improve clinical outcomes.

CMR-FT has been proposed as an alternative method to quantify the myocardial strain calculated by post processing the conventional cine magnetic resonance imaging $[16,22]$. Yu et al [23] performed that LV myocardial strain in patients with dilated cardiomyopathy can be sensitively detected by CMR-FT. Similar to the study by Yu et al., we research showed that GPRS, GPCS, and GPLS were significantly reduced in patients with NIDCM combined with VAs, suggesting global LV myocardial deformation impairment in all directions. Additionally, in this study, our research also evaluated the LV strains rate of NIDCM patients with VAs using CMR-FT. Here, we revealed that NIDCM patients with VAs was associated with a significant impairment of myocardial deformation rate in all directions, indicating that LV function relies on coordinated wall motion from all directions.

Consistent with previous research by $\mathrm{Yu}$, et al., in this study, we observed that the degree of damage was not same in each direction. However, surprisingly, in contrast to other work [23], our research found that GPCS, rather than GPRS changed more than GPRS and GPLS, indicating that patients with NIDCM combined with VAs had more serious damage in the circumferential direction of the LV. Some differences in patients' characteristics could explain this discordance. For example, our research objects for patients with NIDCM combined with VAs while Yu's subjects were only just DCM. Because in this study, we showed that late gadolinium enhancement (LGE) was present in 29 (85\%) of largely patients with NIDCM combined with VAs. In addition, previous studies have shown that LGE is the substrate for the occurrence of VAs [24], and VAs mainly occur in the areas of myocardial fibrosis of the dilated myocardium of NIDCM. Furthermore, several other studies proved that classical NIDCM showed the LGE positivity in nonischemic patterns with involvement of areas subjected to increased tension, such as the interventricular septal mid-wall at the site of insertion of ventricular fibers $[25,26]$; it is mostly influenced by circumferentially oriented muscle fibers in the mid-wall. Since NIDCM shows typical mid-wall fibrosis on CMR, it more affects the myocardial circumferential strain, which leads to a decrease in GPCS in patients with NIDCM combined with VAs. All of these changes can lower the middle-wall or epicardial oxygen demand and increase the middlewall and epicardial blood flow, finally leading to reduced peak stains (GPCS, GPRS, and GPLS) in patients with NIDCM combined with VAs.

Additionally, for the segmental myocardial strains, our research demonstrated that the PCS, PRS, PLS, PCSR, PRSR, and PLSR were significantly decreased in most LV myocardial segments, but the intensities of different strains were widely different from one another. This is concomitant with the truth that NIDCM is characterized as diffuse progression over all the whole heart; however, the degree of impairment was heterogeneous.

The LVEF is used as an important marker with which to assess LV function. Regarding the correlation between the LV global strain values and LVEF for patients with NIDCM, only a few studies have been published [23,27]. They found that the LV myocardial strains were significantly associated with the LVEF. Similar to previous studies [23, 27], in this study, we proved that the LV myocardial strains and myocardial strains rate were significantly associated with the LVEF. The reason may be as follows. Myocardial strain measures the degree of deformation of a myocardial segment from its initial length (usually in end-diastole) to its maximum length (usually in end-systole) and is expressed as a percentage. Thus, the strain determines the fractional change in the one-dimensional length from end-diastole to end-systole [28], and the LVEF reflects the fractional change in the three-dimensional volume; therefore, these two measures are in parallel, which may lead to the significant correlation between the myocardial strain and the LVEF. Moreover, in this study, we showed that GPCS was better negatively correlated with LVEF $(r=-0.946, P<0.001)$, and proved that the higher reproducibility intraobserver and interobserver ICC were respectively 0.96 and 0.96 for GPCS measurements, indicating GPCS was a feasible feature tracking parameter in detecting patients with NIDCM combined with VAs. Because the GPCS was not affected by poor tracking of the subannular region, unlike GPLS. For this reason, it was considered the most robust parameter in CMR-FT studies of myocardial strain.

Furthermore, regarding the correlations among the LV global strain values for patients with NIDCM, only a few studies have been published $[23,29]$. Maciver. et al. investigated that the GPCS had a linear correlation with the GPLS by three-dimensional speckle tracking echocardiography [29]. And, Yu, et al showed linear correlations among GPRS, GPCS, and GPLS one another [23]. In this present research, we carried out pair-wise contrast among the GPRS, GPCS, and GPLS, which demonstrated linear correlations among 
one another. Moreover, we showed pair-wise comparison among the GPRSR, GPCSR, and GPLSR, which proved linear correlations among one another (Fig. 3). These consequences suggested that the generation of LV global myocardial strain and strain rate were based on integrated rather than isolated.

\section{Limitations}

Firstly, this was a single-center observational study with a limited number of patients with NIDCM combined VAs. Records were retrospectively collected and diagnosed for NIDCM combined with VAs. Secondly, lots of different factors may influence the quantification of LV strain, including image acquisition, algorithms and even CVI software versions [30]. The strains values supplied by our study are algorithms and software specific, they can't be simply used under other circumstances.

\section{Conclusions}

In conclusion, LV global strains assessed by CMR-FT imaging could identify patients with NIDCM combined VAs and healthy volunteers. And, GPCS was better negatively correlated with LVEF and higher reproducibility of intra-class correlation coefficient (ICC), suggesting it was the feasible feature tracking parameter in detecting patients with NIDCM combined with VAs. These findings have important implications for the design of future studies evaluating the role of VAs risk stratification in patients with NIDCM combined VAs.

\section{Declarations}

\section{Conflict of interest}

The authors declare that they have no conflicts of interest.

\section{Funding}

This study was supported by the Science and Technology Foundation of the Health Commission of Guizhou province (Grant No.gzwjkj 2020-1-176), Guizhou provincial people's hospital doctoral program (Grant No. GZSYBS[2018]09).

\section{References}

1. Kadish A, Dyer A, Daubert JP, Quigg R et al (2004) Defibrillators in Non-Ischemic Cardiomyopathy Treatment Evaluation I Prophylactic defibrillator implantation in patients with nonischemic dilated cardiomyopathy. N Engl J Med. 350(21):2151-2158

2. Bardy GH, Lee KL, Mark DB et al (2005) Sudden Cardiac Death in Heart Failure Trial I Amiodarone or an implantable cardioverterdefibrillator for congestive heart failure. N Engl J Med. 352(3):225-237

3. Arbustini E, Narula N, Dec GW et al (2013) The MOGE(S) classification for a phenotype-genotype nomenclature of cardiomyopathy: endorsed by the World Heart Federation. J Am Coll Cardiol. 62(22):2046-2072

4. Felker GM, Thompson RE, Hare JM et al (2000) Underlying causes and long-term survival in patients with initially unexplained cardiomyopathy. N Engl J Med. 342(15):1077-1084

5. Jefferies JL, Towbin JA (2010) Dilated cardiomyopathy. Lancet. 375(9716):752-762

6. Neilan TG, Coelho-Filho OR, Danik SB et al (2013) CMR quantification of myocardial scar provides additive prognostic information in nonischemic cardiomyopathy. JACC Cardiovasc Imaging. 6(9):944-954

7. Halliday BP, Gulati A, Ali A et al (2017) Association Between Midwall Late Gadolinium Enhancement and Sudden Cardiac Death in Patients With Dilated Cardiomyopathy and Mild and Moderate Left Ventricular Systolic Dysfunction. Circulation. 135(22):21062115

8. Piers SR, Everaerts K, van der Geest RJ et al (2015) Myocardial scar predicts monomorphic ventricular tachycardia but not polymorphic ventricular tachycardia or ventricular fibrillation in nonischemic dilated cardiomyopathy. Heart Rhythm. 12(10):2106- 
9. Strohm O, Schulz-Menger J, Pilz B et al (2001) Measurement of left ventricular dimensions and function in patients with dilated cardiomyopathy. J Magn Reson Imaging. 13(3):367-371

10. Japp AG, Gulati A, Cook SA et al (2016) The Diagnosis and Evaluation of Dilated Cardiomyopathy. J Am Coll Cardiol. 67(25):2996-3010

11. Porcari A, De Angelis G, Romani S et al (2019) Current diagnostic strategies for dilated cardiomyopathy: a comparison of imaging techniques. Expert Rev Cardiovasc Ther. 17(1):53-63

12. Plana JC, Galderisi M, Barac A et al (2014) Expert consensus for multimodality imaging evaluation of adult patients during and after cancer therapy: a report from the American Society of Echocardiography and the European Association of Cardiovascular Imaging. Eur Heart J Cardiovasc Imaging 15:1063-1093

13. Celutkiene J, Plymen CM, Flachskampf FA et al (2018) Innovative imaging methods in heart failure: a shifting paradigm in cardiac assessment. Position statement on behalf of the Heart Failure Association of the European Society of Cardiology. Eur $\mathrm{J}$ Heart Fail 20:1615-1633

14. Bistoquet A, Oshinski J, Skrinjar O (2008) Myocardial deformation recovery from cine MRI using a nearly incompressible biventricular model. Med Image Anal. 12(1):69-85

15. Grothues F, Smith GC, Moon JC et al (2002) Comparison of interstudy reproducibility of cardiovascular magnetic resonance with two-dimensional echocardiography in normal subjects and in patients with heart failure or left ventricular hypertrophy. Am J Cardiol. 90(1):29-34

16. Obokata M, Nagata Y, Wu VC et al (2016) Direct comparison of cardiac magnetic resonance feature tracking and 2D/3D echocardiography speckle tracking for evaluation of global left ventricular strain. Eur Heart J Cardiovasc Imaging. 17(5):525-532

17. Pinto YM, Elliott PM, Arbustini E et al (2016) Proposal for a revised definition of dilated cardiomyopathy, hypokinetic non-dilated cardiomyopathy, and its implications for clinical practice: a position statement of the ESC working group on myocardial and pericardial diseases. Eur Heart J. 37(23):1850-1858

18. Kramer CM, Barkhausen J, Bucciarelli-Ducci C et al (2020) Standardized cardiovascular magnetic resonance imaging (CMR) protocols: 2020 update. J Cardiovasc Magn Reson. 22(1):1-18

19. Moore CC, Lugo-Olivieri CH, McVeigh ER et al (2000) Three-dimensional systolic strain patterns in the normal human left ventricle: characterization with tagged MR imaging. Radiology. 214(2):453-466

20. Cerqueira MD, Weissman NJ, Dilsizian V et al (2002) American Heart Association Writing Group on Myocardial S, Registration for Cardiac I Standardized myocardial segmentation and nomenclature for tomographic imaging of the heart. A statement for healthcare professionals from the Cardiac Imaging Committee of the Council on Clinical Cardiology of the American Heart Association. Circulation. 105(4):539-542

21. Hamdan A, Thouet T, Kelle S et al (2009) Strain-encoded MRI to evaluate normal left ventricular function and timing of contraction at 3.0 Tesla. J Magn Reson Imaging. 29(4):799-808

22. Ohyama Y, Ambale-Venkatesh B, Chamera E et al (2015) Comparison of strain measurement from multimodality tissue tracking with strain-encoding MRI and harmonic phase MRI in pulmonary hypertension. Int J Cardiol. 182(7):342-348

23. Yu Y, Yu S, Tang X et al (2017) Evaluation of left ventricular strain in patients with dilated cardiomyopathy. J Int Med Res. 45(6):2092-2100

24. Schmidt A, Azevedo CF, Cheng A et al (2007) Infarct tissue heterogeneity by magnetic resonance imaging identifies enhanced cardiac arrhythmia susceptibility in patients with left ventricular dysfunction. Circulation. 115(15):2006-2014

25. McCrohon JA, Moon JC, Prasad SK et al (2003) Differentiation of heart failure related to dilated cardiomyopathy and coronary artery disease using gadolinium-enhanced cardiovascular magnetic resonance. Circulation. 108(1):54-59

26. de Leeuw N, Ruiter DJ, Balk AH et al (2001) Histopathologic findings in explanted heart tissue from patients with end-stage idiopathic dilated cardiomyopathy. Transpl Int. 14(5):299-306

27. Cheng-Baron J, Chow K, Pagano JJ et al (2015) Quantification of circumferential, longitudinal, and radial global fractional shortening using steady-state free precession cines: a comparison with tissue-tracking strain and application in Fabry disease. Magn Reson Med. 73(2):586-596 
28. Scatteia A, Baritussio A, Bucciarelli-Ducci C (2017) Strain imaging using cardiac magnetic resonance. Heart Fail Rev. 22(4):465476

29. Maciver DH (2012) The relative impact of circumferential and longitudinal shortening on left ventricular ejection fraction and stroke volume. Exp Clin Cardiol. 17(1):5-11

30. Yang-Yang Qu JP, Hao Li, Gen-Shan Ma et al (2021) Left ventricular myocardial strain quantification with two-and threedimensional cardiovascular magnetic resonance based tissue tracking. Quant Imaging Med Surg. 11(4):1421-1436

Figures
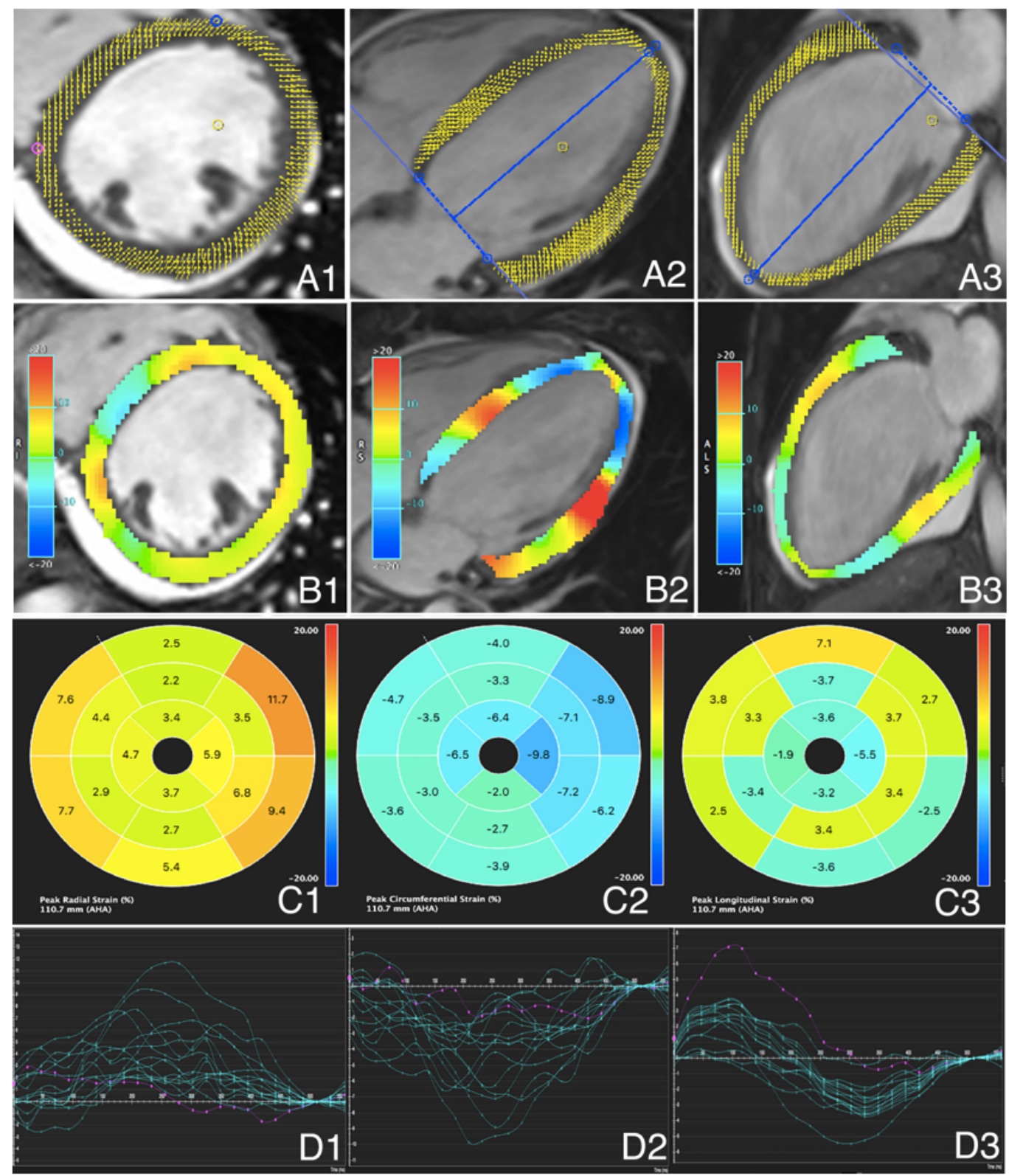

\section{Figure 1}

Representative example of the derivation of strain using cvi42 software in a 44-year-old male patient with NIDCM combined VAs. The result of LV tissue-tracking of cine SSFP images in the short axi, 2-chamber, and 4-chamber view. Picture A1-3 Myocardial strain tissue-tracking are illustrated on short-axis plane, in 4-chamber, and 2-chamber view. Picture B1-3: Myocardial pseudo-color map shows the strain values in the axi, 4- chamber, and 2- chamber view. Picture C1-3: Strain of each segment on the bull's eye diagram of AHA for the peak radial strain (\%), peak circumferential strain (\%), and peak longitudinal strain (\%), Picture D1-3: AHA segmentation 
strain curve values for the radial strain (\%), circumferential strain (\%), and peak longitudinal strain (\%). Red represents positive values, while blue represents negative; deeper colors indicate higher values
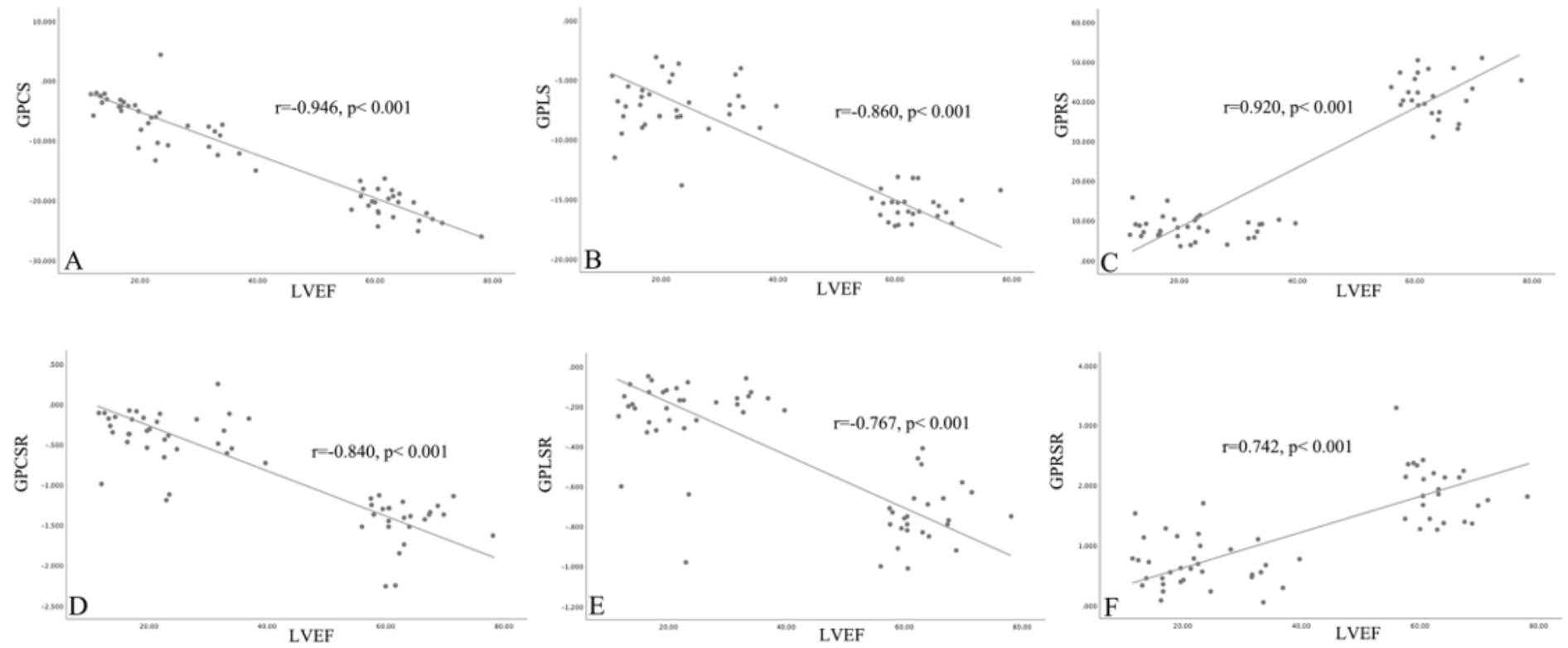

\section{Figure 2}

Pearson analysis suggested that GPCS negatively correlated with LVEF (A), GPLS negatively correlated with LVEF (B), and GPRS positively correlated with LVEF (C); And Pearson analysis also showed that GPCSR negatively correlated with LVEF (D), GPLSR negatively correlated with LVEF (E), and GPRSR positively correlated with LVEF (F)
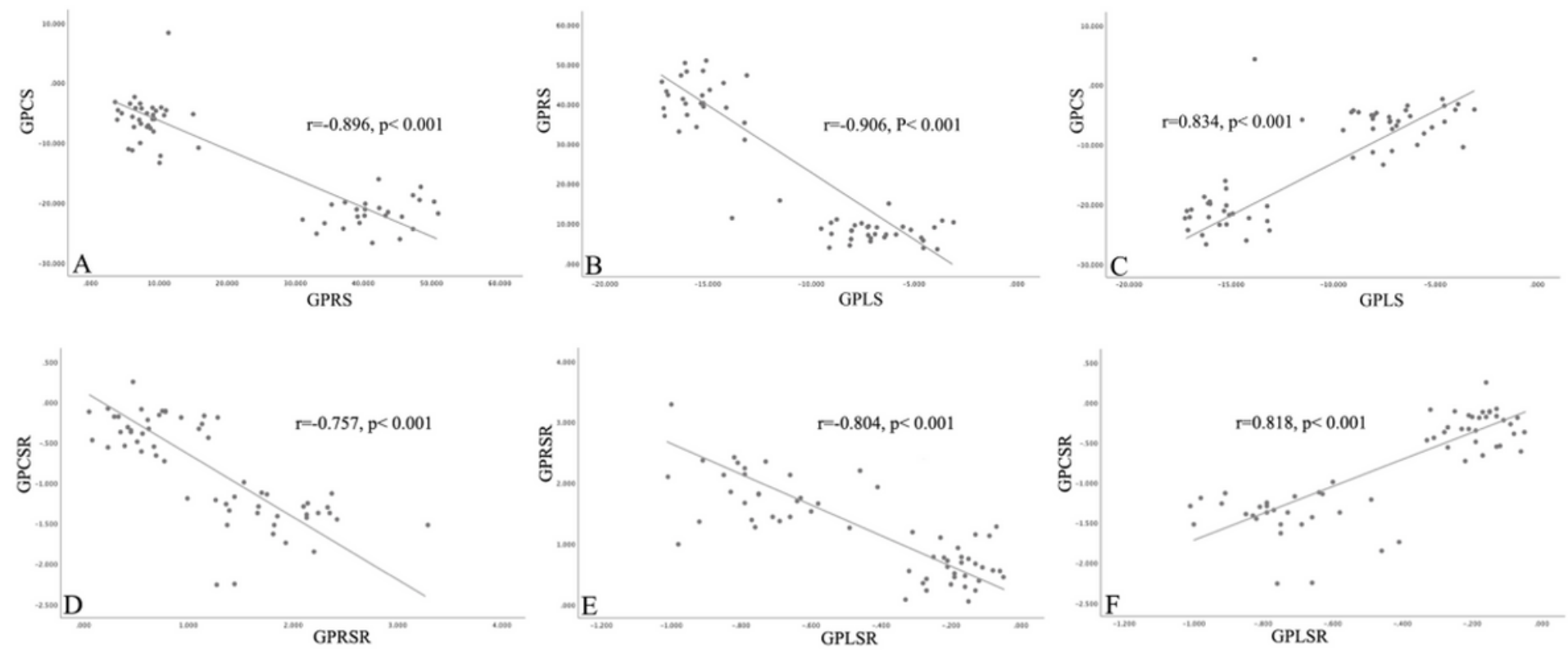

Figure 3

Pearson analysis suggested that GPCS negatively correlated with GPRS (A), GPRS negatively correlated with GPLS (B), and GRCS positively correlated with GPLS (C); Pearson analysis also showed that GPCSR negatively correlated with GPRSR (D), GPRSR negatively correlated with GPLSR (E), and GRCS positively correlated with GPLSR (F) 